\title{
Treatment of Myofascial Pain syndrome in a Hemiplegic Patient with Spasticity: Killing Two Birds with One Stone
}

The Editor,

Sir,

A 44-year old woman with left hemiplegia was seen during a follow-up visit for post-stroke spasticity. She had left hemiplegia caused by a thrombotic cerebrovascular accident in 2009. For spasticity treatment, oral antispasticity drugs (baclofen and tizanidin) were prescribed and botulinum toxin (BTX) injections have been administered for a minimum sixmonth period. Nevertheless, it was difficult to resolve the spasticity in the left-side (especially in the upper limb).

In physical examination, there were distinct stiffness and local pain with palpation in the left trapezius muscle (the patient was diagnosed as myofascial pain syndrome (MPS). Visual analog scale (VAS) for pain was 7/10. She had severe spasticity (modified Ashworth scale [MAS] grade 3) on the left arm (ie shoulder adductors, elbow flexors, forearm pronators and finger flexors). Laboratory evaluations were all normal. Shoulder radiograph was normal and shoulder ultrasonography yielded heterogeneous supraspinatus tendon on the leftside.

Before the BTX injection to the spastic muscles, it was planned to relieve pain of the left trapezius muscle with local anaesthetic injection to the trigger points. One day after injection, the pain on the left trapezius muscle and also the spasticity on the left arm muscles (especially elbow flexors and forearm pronators) were decreased (VAS: 3/10 and MAS: 1). After one week, spasticity of relevant muscles was still at MAS grade 1.

Spasticity is a common activity-limiting complication of stroke (1). Factors such as; urinary tract infections, bladder distension, constipation, anal fissures, haemorrhoids, muscle tears, joint contractures, pressure ulcers and deep vein thrombosis can increase remaining spasticity (2). Stiffness of "nonspastic" muscles leads to decrease in the range of movement that may increase passive stiffness and shortening of the intrafusal fibres. Shortening of the intrafusal fibres of the muscle spindles would result in increased motor output that lead to increase in spasticity (2).

Myofascial pain syndrome is a non-inflammatory musculoskeletal disorder, associated with local pain and muscle stiffness, characterized by the presence of hyperirritable palpable nodules in the muscle fibres, which are named trigger points (3). Several therapeutic approaches such as; spray-andstretch, massage/ischaemic compression, and application of heat are available for MPS, but the gold standard of treatment is local needle penetration - with or without injection of local anaesthetics - to trigger points (4).

We caution clinicians to keep in mind that strained muscles can enhance spasticity in patients with stroke and reducing the stiffness of the muscles can result in decreasing the spasticity. To the best of our knowledge, this is the first case of reducing the post-stroke upper limb spasticity after MPS treatment in the hitherto literature.

Keywords: Hemiplegia, injection, myofascial pain syndrome, spasticity, stroke

\section{S Yalçın, P Çam, SÖ Demir}

From: Ankara Physical Medicine and Rehabilitation Training and Research Hospital, Ankara, Turkey

Correspondence: S Yalçın, Ankara Physical Medicine and Re-habilitation Training and Research Hospital, Ankara, Turkey. Fax: +903123118054,

e-mail:suhayalcin@yandex.com

\section{REFERENCES}

1. Francisco GE, McGuire JR: Poststroke spasticity management. Stroke 2012; 43: 3132-6.

2. Phadke CP, Balasubramanian CK, Ismail F, Boulias C. Revisiting physiologic and psychologic triggers that increase spasticity. Am J Phys Med Rehabil 2013; 92: 357-69.

3. Vázquez-Delgado E, Cascos-Romero J, Gay-Escoda C: Myofascial pain syndrome associated with trigger points: a literature review. (I): Epidemiology, clinical treatment and etiopathogeny. Med Oral Patol Oral Cir Bucal 2009; 14: e494-8.

4. Affaitati G, Fabrizio A, Savini A, Lerza R, Tafuri E, Costantini R. A randomized, controlled study comparing a lidocaine patch, a placebo patch, and anesthetic injection for treatment of trigger points in patients with myofascial pain syndrome: evaluation of pain and somatic pain thresholds. Clin Ther 2009; 31: 705-20. 